\title{
Blood vessel growth and endothelial cell density in rat endometrium
}

\author{
A. M. Goodger (Macpherson) and P. A. W. Rogers \\ Monash University, Department of Obstetrics and Gynaecology, Monash Medical Centre, \\ 246 Clayton Road, Clayton 3168, Australia
}

\begin{abstract}
The aim of the present study was to quantify endothelial cell density in rat endometrium during early pregnancy (a time of increased endothelial cell proliferation), using immunohistochemical methods. Despite the occurrence of vessel growth, the endometrial endothelial cell density remained unchanged before embryo implantation, indicating that endothelial cell proliferation keeps pace with proliferation of the surrounding endometrial cells. After implantation, endothelial cell density falls at embryo sites on day 6, despite very high endothelial cell proliferation rates. This is probably due to the oedema caused by the localized increase in vascular permeability at implantation sites. We hypothesize that this reduction may provide a stimulus for the observed endothelial cell proliferation, rather than a direct angiogenic stimulus from the embryo.
\end{abstract}

\section{Introduction}

In rats and many other eutherian mammals, despite the differing modes of embryo implantation among this diverse group of animals, the earliest sign of embryo implantation is a localized increase in vascular permeability leading to tissue oedema. The underlying physiology of this response is, however, still unknown, although it has been hypothesized that this increased swelling may be a stimulus for mitosis of the surrounding stromal cells and uterine growth (Rogers et al., 1982a). It is presumed that growth of the endometrial stroma is accompanied by vascular growth. We have already shown that in the rat uterus during early pregnancy, the endothelial cell proliferative index increases throughout the entire endometrium from day 3, and then continues at embryo sites alone after implantation (Goodger (Macpherson) and Rogers, 1993). Previously, it has been assumed that an increase in vascular density alone is enough to indicate that angiogenesis or new vascular growth has occurred. The aim of the present study was to quantify endothelial cell density from the same animals and tissue sections. We showed that, despite the occurrence of endothelial cell proliferation and presumed vessel growth, the endometrial endothelial cell density remains unchanged before embryo implantation, and in fact falls at embryo sites immediately after implantation.

\section{Methods}

The methods used were as described in Goodger (Macpherson) and Rogers (1993). Briefly, the uteri from day 1 to day 7 pregnant Sprague-Dawley rats $(n=4$ per day; day $1=$ the day on which spermatozoa were present in the vaginal smear) were perfusion fixed with $10 \% \mathrm{v}$ buffered formalin, divided into pieces, and embedded in wax (Paraplast X-TRA; Oxford

Received 22 May 1995
Labware, St Louis, MO). On days 6 and 7 of pregnancy (that is, after implantation has occurred) uteri were divided into embryo sites and intersites. Proliferating endothelial cells and total numbers of endothelial cells $\mathrm{mm}^{-2}$ (i.e. endothelial cell density) were quantified in approximately consecutive, $5 \mu \mathrm{m}$ sections. For each rat, four blocks (or eight for rats after implantation) were used. In all sections, endothelial cells were identified with a streptavidin-biotin method of immunohistochemistry, using a biotinylated lectin BS-1 from Griffonia (Bandeiraea) simplicifolia (Sigma Chemical Co., St Louis, MO). This lectin has been used as an endothelial cell marker in mice and rats (Laitinen, 1987; Hansen-Smith et al, 1988). It was used at $50 \mu \mathrm{g} \mathrm{ml}^{-1}$, incubated for $30 \mathrm{~min}$ at room temperature, after trypsin (Sigma Chemical Co.) digestion for $10 \mathrm{~min}$ at $37^{\circ} \mathrm{C}$. Immunostaining reagents used were from Zymed Laboratories Inc. (San Francisco, CA). Haematoxylin was used to stain all cell nuclei and, in separate sections, proliferating cells were identified using mouse-anti-rat-proliferating cell nuclear antigen (PCNA; clone PC10; Novocastra Laboratories, Newcastle upon Tyne). The antibody was diluted 1:50 and incubated for $60 \mathrm{~min}$ at $37^{\circ} \mathrm{C}$. Appropriate negative controls were also carried out.

\section{Results}

The lectin used to stain endothelial cells does not stain endothelial cells exclusively (Laitinen, 1987). Non-endothelial cell binding in the present study, however, was not significant enough to interfere with identification of endothelial cells in rat endometrium. Only the apical edges of luminal epithelial cells appeared to bind the lectin from day 4 of pregnancy. Binding of the lectin to decidual cells, however, was more obvious. Blood vessels are known to be absent from the primary decidual zone (Rogers et al., 1982b), so this did not present a problem. However, blood vessels are present in the secondary decidual zone that has developed by day 7 , but were readily distinguishable from decidual cells, as were endothelial cells. 
Table 1. Endothelial cell (EC) density $\left(\mathrm{mm}^{-2}\right)$ and endothelial cell proliferative index (Prl index) for the endometrium of each rat, with mean \pm SEM for each group. Total numbers of endothelial cell nuclei were counted after identification by immunostaining with a biotinylated lectin from Griffonia simplicifolia, and haematoxylin staining. The nuclei of proliferating endothelial cells were identified by double staining with mouse-anti-rat-proliferating cell nuclear antigen and the lectin. The endothelial cell proliferative index is the number of proliferating endothelial cells $\mathrm{mm}^{-2}$ divided by the total number of endothelial cells $\mathrm{mm}^{-2}$, expressed as a percentage

\begin{tabular}{|c|c|c|c|c|}
\hline $\begin{array}{l}\text { Day of } \\
\text { pregnancy }\end{array}$ & $\begin{array}{l}\text { EC density } \\
\text { per } \mathrm{mm}^{2}\end{array}$ & $\begin{array}{c}\text { Group } \\
\text { mean } \pm \text { SEM }\end{array}$ & $\begin{array}{c}\text { EC Prl index } \\
(\%)\end{array}$ & $\begin{array}{c}\text { Group } \\
\text { mean } \pm \text { SEM }\end{array}$ \\
\hline \multirow[t]{4}{*}{1} & 498.53 & $463.24 \pm 19.12$ & 1.28 & $1.04 \pm 0.38$ \\
\hline & 417.86 & & 0.86 & \\
\hline & 445.58 & & 1.91 & \\
\hline & 490.99 & & 0.09 & \\
\hline \multirow[t]{4}{*}{2} & 278.93 & $379.08 \pm 61.36$ & 1.6 & $0.80 \pm 0.28$ \\
\hline & 479.38 & & 0.47 & \\
\hline & 490.99 & & 0.78 & \\
\hline & 267.01 & & 0.34 & \\
\hline \multirow[t]{4}{*}{3} & 696.37 & $484.74 \pm 79.83$ & 7.41 & $13.31 \pm 2.54$ \\
\hline & 485.29 & & 12.33 & \\
\hline & 310.92 & & 13.74 & \\
\hline & 446.39 & & 19.74 & \\
\hline \multirow[t]{4}{*}{4} & 221.04 & $385.97 \pm 71.69$ & 22.03 & $19.71 \pm 2.97$ \\
\hline & 370.49 & & 13.07 & \\
\hline & 570.93 & & 17.03 & \\
\hline & 381.40 & & 26.71 & \\
\hline \multirow[t]{4}{*}{5} & 567.57 & $416.80 \pm 80.74$ & 25.47 & $27.68 \pm 1.96$ \\
\hline & 383.36 & & 28.59 & \\
\hline & 204.03 & & 32.78 & \\
\hline & 512.25 & & 23.88 & \\
\hline \multirow[t]{4}{*}{6 Embryo sites } & 237.54 & $280.44 \pm 20.04$ & 52.14 & $53.59 \pm 5.66$ \\
\hline & 295.72 & & 67.47 & \\
\hline & 328.72 & & 39.86 & \\
\hline & 259.95 & & 54.90 & \\
\hline \multirow[t]{4}{*}{6 Intersites } & 355.35 & $507.25 \pm 61.43$ & 6.17 & $4.21 \pm 1.99$ \\
\hline & 611.62 & & 1.86 & \\
\hline & 602.39 & & 0.03 & \\
\hline & 459.65 & & 8.79 & \\
\hline \multirow[t]{4}{*}{7 Embryo sites } & 316.20 & $358.67 \pm 26.57$ & 60.35 & $71.27 \pm 5.13$ \\
\hline & 431.20 & & 73.22 & \\
\hline & 321.79 & & 84.50 & \\
\hline & 365.48 & & 67.01 & \\
\hline \multirow[t]{4}{*}{7 Intersites } & 542.19 & $537.28 \pm 44.73$ & 0.26 & $0.11 \pm 0.06$ \\
\hline & 409.25 & & 0.00 & \\
\hline & 601.13 & & 0.09 & \\
\hline & 596.55 & & 0.09 & \\
\hline
\end{tabular}

Table I shows the endothelial cell density $\left(\right.$ per $\mathrm{mm}^{2}$ ) and the endothelial cell proliferative index for the endometrium of each rat, with mean \pm SEM for each group, across the first 7 days of pregnancy. Before embryo implantation, the endothelial cell density in the endometrium did not significantly change during the first 5 days of pregnancy (as shown by a one-way ANOVA; $\quad F=0.49 ; \quad P=0.742$ ), despite the significantly increased endothelial cell proliferation over the same period $\left(F=35.68 ; P=1.69 \times 10^{-7}\right)$. After implantation, there is a significant reduction in endothelial cell density on day 6 of pregnancy at embryo sites compared with the first 5 days of pregnancy (data combined; as shown by Student's t-test;
$P=0.034)$. A significant reduction in endothelial cell density also occurred at embryo sites compared with intersites for day 6 of pregnancy (Paired $t$-test; $P=0.014$ ), but not day 7 $(P=0.078)$, despite simultaneous increases in endothelial cell proliferation $(P=0.003$ and $P=0.001$, respectively).

\section{Discussion}

These results show that, even though the endometrial endothelial cell proliferation rate increases steadily from day 3 to day 5 of pregnancy (thereby contributing to vessel growth), 
endothelial cell density remains constant. From day 2 to day 5 , the endometrium is growing, as shown by increased cellular proliferation of the epithelium and stromal cells (Chaudhury and Sethi, 1970; Goodger (Macpherson) and Rogers, 1993). Together these data indicate that in rat endometrium before implantation, vascular growth occurs in concert with overall tissue growth.

At intersites after embryo implantation, endothelial cell density remains unchanged, and stromal and endothelial cell proliferation returns to basal values. At embryo sites on day 6 of pregnancy, the endothelial cell density is significantly reduced compared with the previous days, and compared with intersites. By day 7, the endothelial cell density at embryo sites is not significantly different from that at intersites. This reduction on day 6 at implantation sites, despite the increased endothelial cell proliferation, is likely to be due to the tissue volume expansion caused by the localized increase in microvascular permeability and consequent tissue oedema. This begins from the late afternoon/early evening of day 5 and lasts until at least the afternoon of day 6 . We hypothesize that the tissue volume expansion and stretching, although initially responsible for a reduction in endothelial cell density on day 6 , would, together with the expected reduction in oxygenation, provide further stimuli for continued endometrial blood vessel growth.

The results of this study showed that vessel growth can occur in a tissue without an increase in endothelial cell density, and that vessel growth can occur with a simultaneous decrease in endothelial cell density. The assumption that a measurable increase in endothelial cell or blood vessel density indicates vessel growth, is too simple. The relative rates of cell division or death of the surrounding cells, as well as volume changes in the extracellular matrix, must be considered as well. This assumption has also been shown to be incorrect for the endometrium of users of Norplant. These women have increased endometrial microvascular and endothelial cell densities compared with control women (Rogers et al., 1993; Goodger (Macpherson) et al., 1994) which is not due to increased endothelial cell proliferation (which is significantly reduced), but is likely to be due to a reduced rate of regression of the blood vessels relative to the rest of the endometrial tissue.

A. M. Macpherson was the recipient of a Monash University Postgraduate Writing-Up Award.

\section{References}

Chaudhury RR and Sethi A (1970) Effects of an intra-uterine contraceptive device in the rat uterus on different days of pregnancy Journal of Reproduction and Fertility 22 33-40

Goodger (Macpherson) AM and Rogers PAW (1993) Proliferating endothelial cells in the endometrium of early pregnant rats Journal of Reproduction and Fertility 99 451-457

Goodger (Macpherson) AM, Rogers PAW and Affandi B (1994) Endometrial endothelial cell proliferation in longterm users of subdermal levonorgestrel Human Reproduction 9 1647-1651

Hansen-Smith FM, Watson L, Lu DY and Goldstein I (1988) Griffonia simplicifolia I: fluorescent tracer for microcirculatory vessels in nonperfused thin muscles and sectioned muscle Microvascular Research 36 199-215

Laitinen L (1987) Griffonia simplicifolia lectins bind specifically to endothelial cells and some epithelial cells in mouse tissues Histochemical Journal 19 225-234

Rogers PAW, Murphy CR and Gannon BJ (1982a) Changes in the spatial organization of the uterine vasculature during implantation in the rat Journal of Reproduction and Fertility 65 211-214

Rogers PAW, Murphy CR and Gannon BJ (1982b) Absence of capillaries in the endometrium surrounding the implanting rat blastocyst Micron 13 373-374

Rogers PAW, Au CL and Affandi B (1993) Endometrial microvascular density during the normal menstrual cycle and following exposure to long-term levonorgestrel Human Reproduction 8 1396-1404 